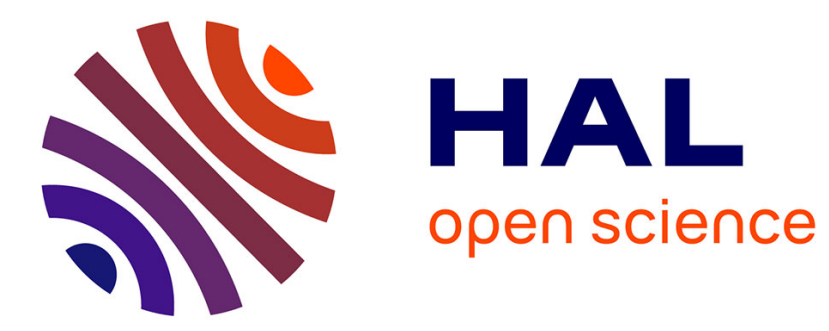

\title{
Time scales for drainage and imbibition in gellified foams. Application to decontamination processes
} Rémi Deleurence, Tamar Saison, François Lequeux, Cécile Monteux

\section{To cite this version:}

Rémi Deleurence, Tamar Saison, François Lequeux, Cécile Monteux. Time scales for drainage and imbibition in gellified foams. Application to decontamination processes. Soft Matter, 2015, 11 (35), pp.7032-7037. 10.1039/C5SM01158B . hal-01198763

\section{HAL Id: hal-01198763 https://hal.sorbonne-universite.fr/hal-01198763}

Submitted on 17 Sep 2015

HAL is a multi-disciplinary open access archive for the deposit and dissemination of scientific research documents, whether they are published or not. The documents may come from teaching and research institutions in France or abroad, or from public or private research centers.
L'archive ouverte pluridisciplinaire HAL, est destinée au dépôt et à la diffusion de documents scientifiques de niveau recherche, publiés ou non, émanant des établissements d'enseignement et de recherche français ou étrangers, des laboratoires publics ou privés. 


\title{
Time scales for drainage and imbibition in gellified foams. Application to decontamination processes.
}

\author{
Rémi Deleurence $^{[a, b]}$, Tamar Saison ${ }^{[c]}$, François Lequeux ${ }^{[a, b]}$ and Cécile Monteux ${ }^{[a, b]}$ *
}

\begin{abstract}
We probe the drainage and imbibition dynamics of foams in which the continuous aqueous phase is a transient gellike network. To produce these foams, we provide a new method - a PVA (Polyvinyl alcohol) solution is first foamed and then a cross-linker, Borax, is added, which binds reversibly to the PVA chains. Ther resulting foams are ultra-stable-over a month. We find that the typical time for gravitational drainage of the continuous phase can be slowed down from hours to several weeks by tuning the Borax concentration. We show that the Borax concentration controls both the bulk viscosity of the continuous phase and the surface viscosity of the air-water interfaces. From these results we suggest that the PVA molecules adsorbed at the bubbles interfaces are highly cross-linked by the Borax molecules. We find that the capillary rise of a dyed liquid into these foams is orders of magnitude faster than the drainage flow, meaning that these foams can quickly absorb liquids. These results show that these foams could be used to clean or decontaminate surfaces covered with liquid wastes. Indeed we show that the PVA-Borax foam can easily be spread on a surface, absorb a liquid without destabilizing and be dried afterward to recover the waste.
\end{abstract}

\section{Introduction}

Aqueous foams, composed of over $90 \%$ in volume of air bubbles in an aqueous phase liquid, are used as precursors for the production of porous lightweight materials ${ }^{12}$, for their acoustic ${ }^{3}$, electric or insulating ${ }^{4}$ properties as well as for cleaning and decontamination purposes $s^{5}$, , as liquids can be trapped in the foam channels by capillary flows ${ }^{7}$. For example, in nuclear reactors, where radioactive wastes are present on solid surfaces, liquid foams are promising materials to absorb the wastes and clean surfaces as they enable to reduce the quantity of radioactive liquid to be decontaminated or stored afterwards, which is a crucial issue. However, conventional surfactant foams have a limited life-time - minutes - as gravity induces a drainage flow of the aqueous phase which destabilizes the foam ${ }^{8,9}$.

To obtain highly stable foams, one possible strategy is to increase the viscosity of the aqueous phase, to decrease the rate of gravitational drainage, which tends to destabilize the foams. Several studies report the use of complex yield stress fluids such as Laponite ${ }^{10}$ or flocculated suspensions ${ }^{11}$. Synthesis of porous scaffold materials based on gellified foams made with alginate ${ }^{12,13}$ or gelatin $^{14,15}$ have been reported in the scientific literature, however there are very few fundamental studies devoted to their stability. Moreover, producing large quantities of viscous foams remains a

a) École Supérieure de Physique et de Chimie Industrielles de la Ville de Paris (ESPCI), ParisTech, PSL Research University, Sciences et Ingénierie de la Matière Molle (SIMM), CNRS UMR 7615, 10 rue Vauquelin, F-75231 Paris cedex 05, France.

b) Sorbonne-Universités, UPMC Univ Paris 06, SIMM, 10 rue Vauquelin, F-75231 Paris cedex 05, France.

c) Saint-Gobain recherche, 39 Quai Lucien Lefranc, 93300 Aubervilliers, France Electronic Supplementary Information (ESI) available: [details of any supplementary information available should be included here]. See DOI: $10.1039 / x 0 x x 00000 x$ technical challenge, as air entrainment and bubble fractionation is difficult in highly viscous liquids ${ }^{11}$. To circumvent this issue, it is easier to first produce the bubbles in a polymer solution and then add the cross-linker to obtain the gelation of the aqueous phase, as was done for covalently-bonded gels ${ }^{15,16}$ in a microfluidic device.

In this article, we provide a new and simple method to produce large quantities - liters - of highly stable foams - over a month where the aqueous phase is a gel-like transient network, where polymer chains, PVA, poly vinylalcohol, bind to Borax molecules through reversible links. Unlike covalently-bonded polymer gels, which are fragile and tend to fracture easily, PVA/Borax materials can be more easily deformed and hence processed as the complexes are reversible. These foams have outstanding properties: they are ultra-stable -over a month- and they can quickly absorb liquids. We find that the rate of drainage can be tuned over orders of magnitude by the Borax concentration, which controls both the bulk and interfacial viscosities. Indeed, our drainage data suggest that increasing the Borax concentration leads to high surface viscosities of the PVA adsorbed layers, meaning that the PVA chains are cross-linked not only in the bulk solution but also at the air-water interfaces. Besides, we probe the capillary rise of a dyed liquid into these foams and find that the imbibition dynamics is orders of magnitude faster than the drainage flow. These foams are therefore good candidates for cleaning or decontamination applications.

\section{Experimental}

A Materials Sodium tetraborate decahydrate $\mathrm{Na}_{2} \mathrm{~B}_{4} \mathrm{O}_{7} \cdot 10 \mathrm{H}_{2} \mathrm{O}$, or Borax, is purchased from Aldrich and used without further purification. 
Table 1. PVA molecules used

\begin{tabular}{|l|l|l|l|}
\hline Product name & $\begin{array}{l}\text { DHydrol } \\
\text { ysis } \\
(\mathbf{m o l} \%)\end{array}$ & $\begin{array}{l}\mathbf{M}_{\mathbf{w}} \\
\mathbf{( \pm 5 0 0 g / m o l )}\end{array}$ & PDI \\
\hline Mowiol $^{\circledR} 4-88$ & 88 & 28000 & 1.38 \\
\hline Mowiol $^{\circledR} 8-88$ & 88 & 51000 & 1.76 \\
\hline Mowiol $^{\circledR} 10-98$ & 98 & 53000 & 1.52 \\
\hline
\end{tabular}

The PVA solutions with concentrations ranging from 2.5 to $15 \mathrm{wt} \%$ are heated to $85^{\circ} \mathrm{C}$ under vigorous stirring during 3 hours to reach total polymer dissolution, and then cooled to room temperature before use. For all PVA/Borax mixtures, Borax solutions are added to a tenfold greater volume of PVA solution, so that final Borax concentrations are approximately ranging from 0.1 to $0.5 \mathrm{wt} \%$.

\section{B Foam preparation.}

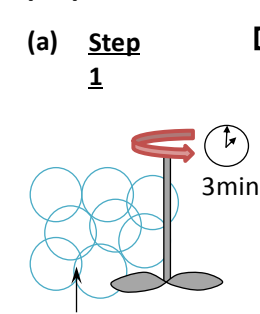

PVA foam

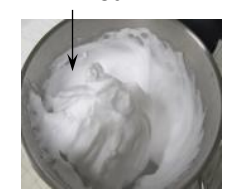

Air Fraction 95\%

Bubble size $\sim 400 \mu \mathrm{m}$

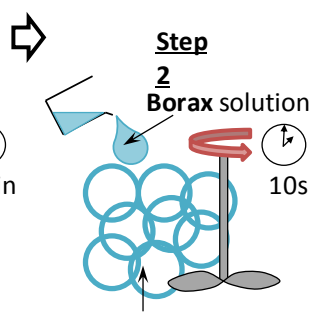

Gellified foam

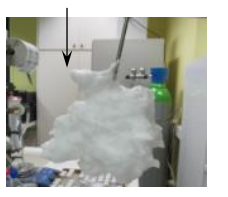

Air Fraction 95\% Bubble size $\sim 400 \mu \mathrm{m}$ (b)

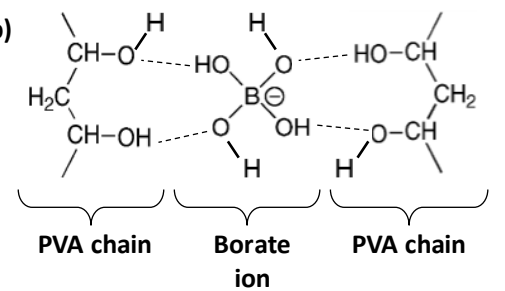

Figure 1. a) 2-step foaming process: a PVA solution is foamed using a mixer and Borax is then added to the foam a) Borate anion can form a complex with two PVA chains and create a transient crosslink.

$200 \mathrm{~g}$ of PVA, (poly(vinyl alcohol) solution is placed into the bowl of a kitchen mixer (Kenwood ${ }^{\circledR}$ KWY90) and sheared (Figure 1a) at a maximal speed $\left(100 \mathrm{~s}^{-1}\right)$ during 3 minutes. PVA, which is a surface active polymer - surface tension around $45 \mathrm{mN} / \mathrm{m}$ - is indeed a very good foaming agent and we obtain 3 to $4 \mathrm{~L}$ of low density fine foam (bubble size $400 \mu \mathrm{m}$, volume fraction of air from 93 to $95 \%$ ).

After the PVA foam is obtained, an aqueous solution of sodium tetraborate $\mathrm{NaB}(\mathrm{OH})_{4}$ (Borax) is added to the PVA foam under continuous mixing for about ten more seconds. $\mathrm{B}(\mathrm{OH})_{4}{ }_{4}$ ions, form reversible complexes with the PVA chains ${ }^{17,18}$, (Figure 1b), which enables to form a transient gellike network whose oscillating shear moduli, G', G' and zero-shear viscosity can be varied over orders of magnitude by changing the Borax concentration (SI1). After 10s, the mixer is stopped and the foam is pumped into a glass cylinder, $16.5 \mathrm{~cm}$ long and $3.8 \mathrm{~cm}$ wide. Both ends of the column are closed to prevent foam from drying, and the column is then placed vertically to study foam ageing at room temperature.

The volume liquid fraction $\phi_{l}$ of foams is determined by weighting the mass $m$ of foam filling a plastic Petri dish of known volume $V_{m}$, $\phi_{l}=\frac{m}{\rho_{l} V_{m}}$, with $\rho_{l}$ the foam fluid density (taken as $1000 \mathrm{~g} / \mathrm{L}$ ). Liquid fractions range from 0.04 to $0.08(+/-0,005)$.

C Foam ageing Photographs of the foam sample are analyzed with ImageJ software to determine the time evolution of the total foam volume and of the so-called drained volume, which is the volume of liquid collected bellow of the foam. The bubble size with time is also estimated through the mean bubble diameter observed at the column walls, mid-height of the column, average is performed over 100 to 200 bubbles.

D Measurement of shear viscosity and storage and loss modulii Rheological measurements are carried out with a AR-G2 Rheometer (TA Instruments) using a cone-plate geometry (cone angle $2^{\circ}$, diameter $40 \mathrm{~mm}$, truncation $52 \mu \mathrm{m}$ ). All measurements are made at $25^{\circ} \mathrm{C}$, and with a solvent trap to avoid evaporation.

E Imbibition with dyed liquid A column of foam is fixed vertically in contact with a reservoir of concentrated aqueous Rhodamine $B$ Base solution (Sigma Aldrich). The rise of the dyed liquid is followed by a digital camera (Canon ${ }^{\circledR}$ SX510 HS). Space time plot on a vertical line is then computed by ImageJ software.

\section{Results}

A Stability and drainage dynamics of the foams. For decontamination or absorption applications, the foam has to remain stable during the absorption process, hence one has to first consider the typical time scales for the stability of the foam. 
(a)

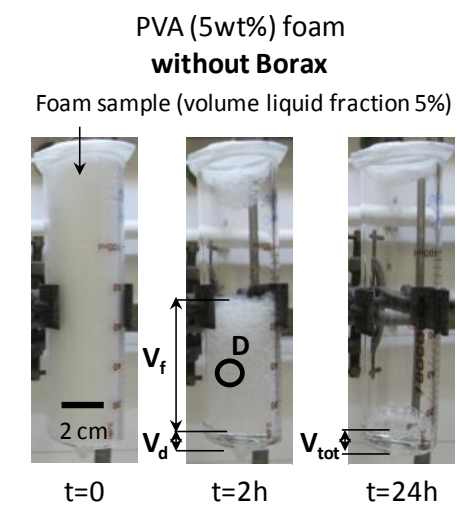

(b)

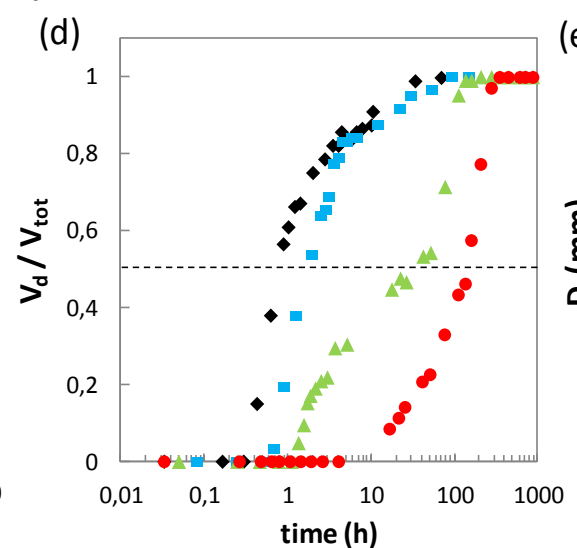

PVA (5wt\%) foam

with Borax 0,4wt\%

Foam sample (volume liquid fraction $5 \%$ )
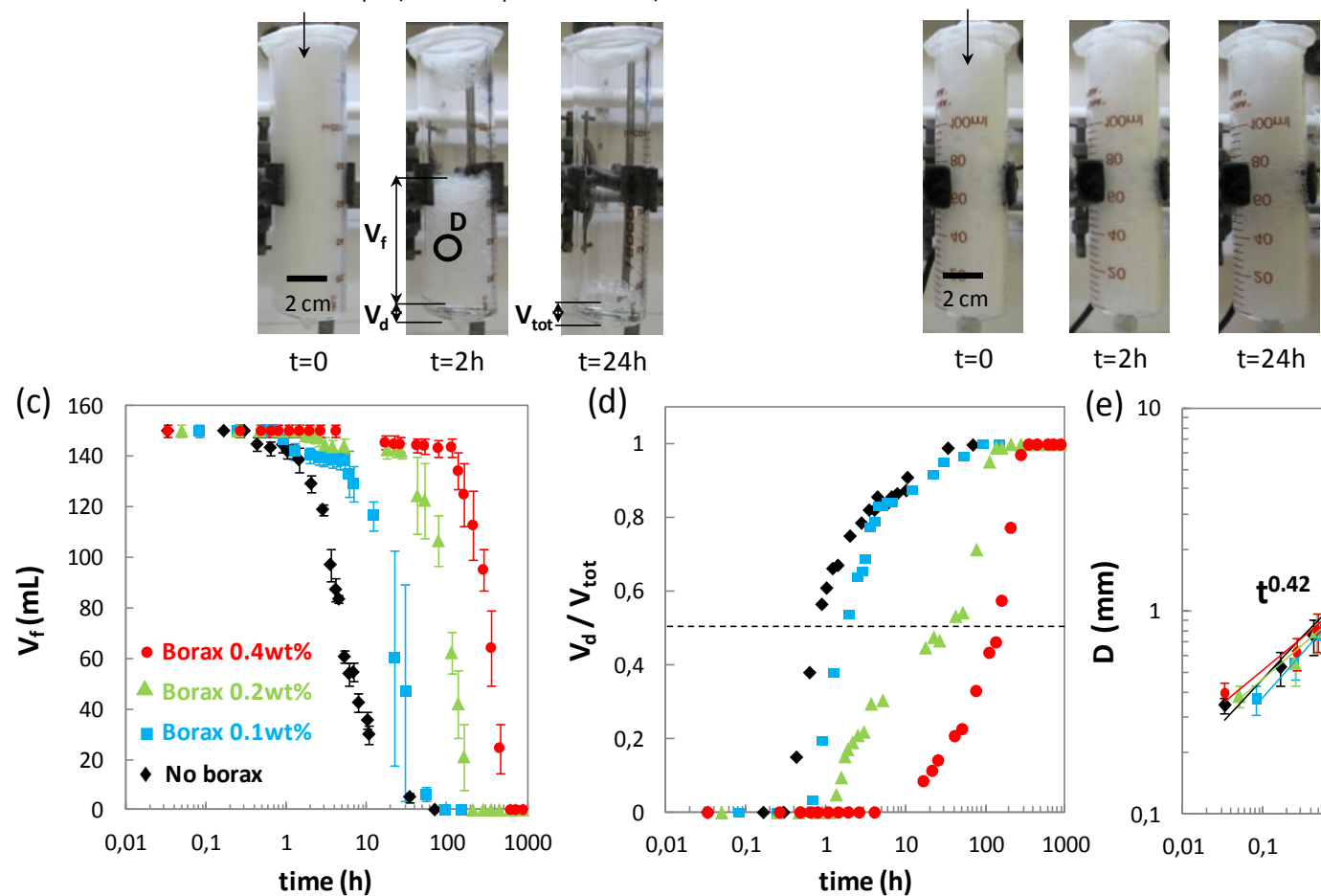

$t=24 h$

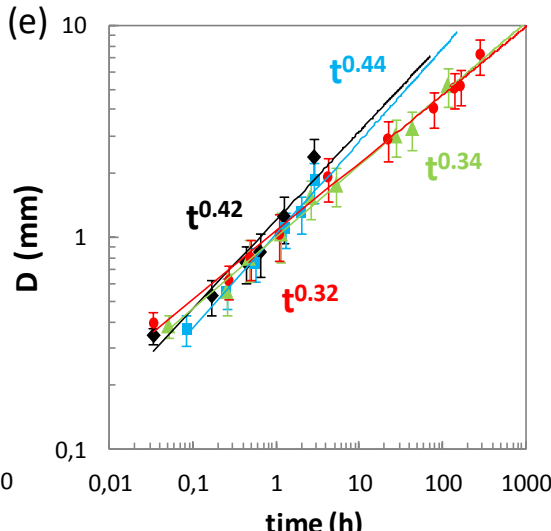

Figure 2. Stability of the PVA/Borax foams. a) and b): Photos of the foam samples as a function of time. The foam without cross-linker destabilizes over a few hours (a) while the Borax-containing foam is stable over several weeks (b). For c), d) and e), the PVA concentration is $5 \mathrm{wt} \%$ and initial liquid volume fraction around 0.05 . The Borax weight percentage is varied between $0 \%$ and $0.4 \mathrm{wt} \% \mathrm{c}$ ) Foam volume, $V_{f}$, as a function of time. d) Volume of drained liquid, $V_{d}$, normalized by total volume of liquid, $V_{t o t}$, as a function of time. Dashed line marks $V_{d} / V_{\text {tot }}=0.5$ corresponding to the half-drainage time $\tau$. e) Diameter of the bubbles at column walls, $D$, as a function of time.

In Figure 2, we report the ageing dynamics of the PVA/Borax foams as a function of the Borax concentration, by measuring the time evolution of the total foam volume, $V_{f}$, the mean bubble diameter at column walls, $D$, and $V_{d} / V_{\text {tot }}$ with $V_{d}$ the volume of liquid collected below of the foam, and $V_{\text {tot }}$ the total volume of liquid in the foam. As the Borax concentration increases from $0 \%$ to $0.4 \%$, the foam stability increases from 10 hours to 500 hours (Figure 2a, $\mathbf{2 b}, \mathbf{2 c}$ and $\mathbf{2 d}$ ). This enhanced stability is driven by the slowing down of the gravity-induced flow of the aqueous phase, so-called drainage flow, that slows down drastically for large Borax concentrations (Figure 2d). Indeed, the half-drainage time $\tau$, defined as the time for half of the liquid to drain, $V_{d} / V_{\text {tot }}=0.5$, increases by up to two orders of magnitude for the Boraxcontaining foams. Besides, at $t=\tau, V_{f}$ does not decrease significantly for these foams (Figure $\mathbf{2 c}$ ) even though a fairly high amount of liquid has drained, meaning that coalescence of the gel films between gas bubbles is negligible.

In addition to drainage and coalescence, a third phenomenon, Ostwald coarsening, i.e diffusion of gas from small bubbles to large bubbles through the aqueous phase, is known to lead to an increase of the bubble size. The Borax concentration does not have a significant influence on the rate of bubble growth (Figure 2e). The Lifshitz-Slyozov-Wagner theory of Ostwald ripening ${ }^{19,20}$ predicts that in the limit of very wet foams, made with a soluble gas, and without drainage, a $t^{1 / 3}$ law is expected for the coarsening kinetics, whereas for very dry foams, it scales as $\mathrm{t}^{1 / 2}$. Considering that our foams are relatively dry (volume liquid fraction $\phi_{l} \sim 0.05$ ), the observed coarsening kinetics scaling between $t^{0.32}$ and $t^{0.44}$ are consistent with predictions. We find that the viscosity has a weak influence on the coarsening dynamics. Indeed, viscosity has been shown to play a role on the coarsening dynamics ${ }^{21}$ only above $10^{7}$ Pa.s, which is above the viscosities of our samples. The weak effect of the viscosity on the coarsening dynamics is probably due to the fact that the gas molecules diffusing from one bubble to another are much smaller than the mesh size of the polymer network. The diffusion of gas is thus not sensitive to the macroscopic viscosity, but only to the one of the solvent, i.e. water.

As explained above, increasing the Borax concentration enables to increase the viscosity of the aqueous phase by orders of magnitude, which slows down the drainage flow. There exist several theoretical models to describe foam drainage and predict the drainage velocity and characteristic time $e^{8,9,22-25}, 6$. Foams are usually considered as a network of channels, known as the Plateau Borders, connected by nodes, and in which the aqueous phase flow is driven by gravity.

The drainage dynamics depends on the boundary conditions at the air-water interface, i.e. whether the amphiphilic layer adsorbed at the air-water interface is mobile and dragged by the flow of the 
aqueous phase. These boundary conditions are set by the Boussinesq number, $B o=\eta_{s} / \eta r$, with $\eta_{s}$ the surface viscosity of the adsorbed layer, $\eta$ the bulk viscosity and $r$ the width of the foam channel. For dry foams, when $B o \gg 1$, the adsorbed layer is considered as 'rigid' and the drainage flow is described by the channel-dominated model. For $B o \lesssim 1$, the node-dominated model describes cases of low surface viscosities and large bulk viscosities.

In the channel-dominated model, the half-drainage time, i.e. the time for which half of the continuous phase has reached the bottom of the foam, scales as

$\tau_{1 / 2}=\frac{Z}{2} \frac{\eta}{\rho_{l} g K_{c}(B o) L^{2} \phi_{l 0}}$, (Equation 1),

where $\mathrm{K}_{\mathrm{c}}(\mathrm{Bo})$ is the non-dimensional permeability factor, which depends on the Boussinesq number and which expression is given in $S \mid 2, L$ is the length of the Plateau borders, $\phi_{l 0}$ the initial volume liquid fraction inside the foam, $\rho_{l}$ the density of the liquid, $g$ the gravity acceleration, $Z$ the foam height $(Z=16.5 \mathrm{~cm})$, and $\eta$ the fluid viscosity.
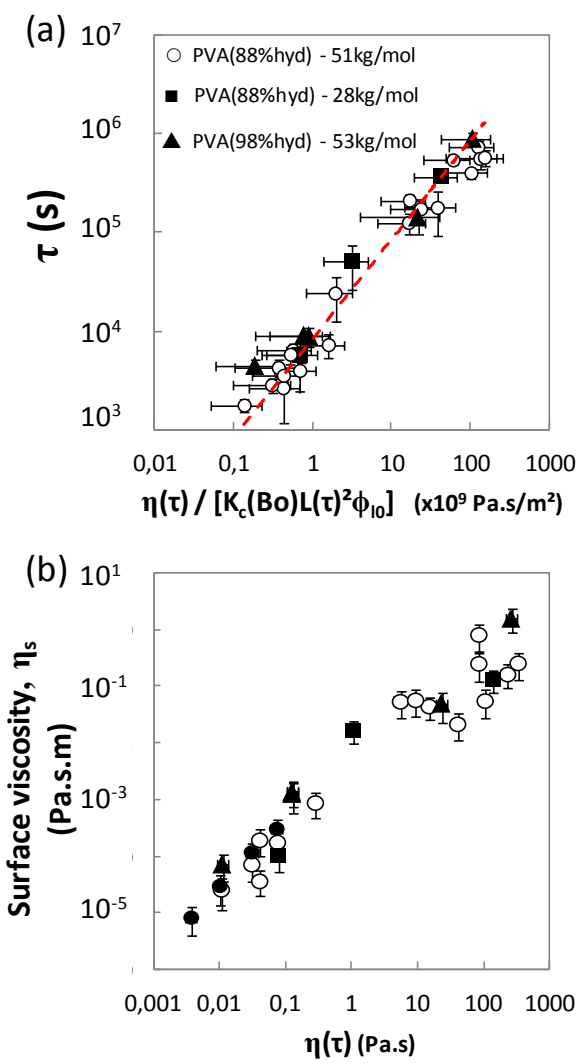

Figure 3. (a) Typical half-drainage time, $\tau$, as a function of the liquid viscosity, $\eta$ for various Borax and PVA concentrations, and PVA with varying molecular weights and degrees of hydrolysis (see experimental section). On the horizontal axis, the viscosity is rescaled with the length of the Plateau borders at $\mathrm{t}=\tau, L(\tau)$, the initial volume liquid fraction $\phi_{10}$, and the permeability coefficient $K_{c}(B o)$. The dotted line represents the fit to Equation 1, the channel dominated regime, taking $B o$ as an adjustable parameter. (b) Values of the surface shear viscosities obtained from the fit to Equation 1.
Figure 3a represents our experimental half-drainage times, $\tau_{1 / 2}$, as a function of the bulk viscosity, $\eta$. To account for the coarseninginduced growth of the bubbles, we replace $L$ by $L(\tau)$. Similarly we replace $\eta$ by $\eta(\tau)$ as the viscosity decreases over time (SI3) due to the hydrolysis of the 2 to $12 \%$ of acetate moieties on the chains ${ }^{27}$. The dotted line represents a fit to Equation 1, taking Bo as an adjustable parameter. From the fitted Bo values, knowing the bulk viscosity $\eta$ and the radius of the Plateau borders, $r$, we can deduce the value of the surface viscosity, $\eta_{s}$, in our foams (Figure 3 ).

Surprisingly, we find that the Boussinesq number remains well above 1 and hence that the channel dominated model is valid over the whole range of bulk viscosities that we investigated. This means that the interfaces can be considered as immobile in all our experiments. We find that the surface viscosities increase from from $10^{-5}$ Pa.s.m to 1 Pa.s.m as the bulk viscosity is increased (Figure 3b). The lowest values are in agreement with measurements done independently using a double wall ring apparatus ${ }^{28}$ for the solutions without Borax (SI4). The largest values, i.e. 1 Pa.s.m, are in agreement with measurements made with highly compressed polymer monolayers ${ }^{29}, 30$.

Therefore increasing the Borax concentration leads to an increase of both the bulk and the surface viscosities. Indeed, before adding the Borax in the foams, the local concentration of PVA chains adsorbed at the air-water interfaces is higher than in the bulk (this is known as the surface excess ${ }^{31}$ ). Therefore after adding the Borax, the PVA layers probably become densely cross-linked with borate ions and hence highly viscous.

B Imbibition dynamics To assess the potential of these foams for decontamination applications, we now probe their absorption dynamics by putting a column of foam in contact with a reservoir of dyed water (Figure 4a). For the PVA foam without Borax (PVA $\left.5 w t \%, \phi_{l 0}=0.071\right)$, as the dyed liquid penetrates into the foam, the foam collapses because the continuous phase is being diluted by the rising liquid, as was already observed by Mensire et $a l^{32}$. As a consequence, after a short rise, the height reaches a maximum and the dyed liquid is dragged back into the reservoir. In the case of the $0.4 w t \%$ Borax foam (PVA $5 w t \%, \phi_{l 0}=0.065$ ), the rise is also very quick but the dye remains in the foam for hours.

Unexpectedly, for a given Borax concentration, the typical time scale for the imbibition kinetics is much shorter than the drainage times reported earlier, which is a promising condition for decontamination applications, where the foam has to remain stable for hours during the imbibition process. This unexpected result can be understood by considering the imbibition dynamics in more details. The imbibition is due to the pressure difference between the water reservoir and the pressure inside the channels due to the curvature radius $r$ of the Plateau borders (Figure $4 \mathrm{~b}$ ). The equilibrium height $h_{\max }$ is reached when the hydrostatic pressure is equilibrated by the capillary pressure, hence $h_{\text {max }} \sim \gamma / r \rho_{l} g$ (Equation 2) where $\gamma \sim 42 \mathrm{mN} / \mathrm{m}$ is the surface tension. Taking for $r$ the typical size of 100 to $200 \mu \mathrm{m}$, we get $h_{\max } \sim 2$ to $4 \times 10^{-2} \mathrm{~m}$, which is consistent with the experimental result (Figure $4 c$ ). The imbibition dynamics is controlled by the balance between viscous 
dissipation of liquid flowing in the channels and the capillary pressure. Assuming rigid interfaces, we can use the Stokes equation in the limit of small Reynolds number, leading to $\frac{\boldsymbol{\eta}}{\boldsymbol{r}^{2}} \frac{\boldsymbol{d} \boldsymbol{h}}{\boldsymbol{d} \boldsymbol{t}}=\frac{\gamma / \boldsymbol{r}}{\boldsymbol{h}}-\rho_{l} g$ (Equation 3) ${ }^{33}$.

Figure 4 shows that a very good agreement between our experiments and the numerical resolution of Equation 3 , is obtained for $r=150 \mu \mathrm{m}, \gamma=42 \mathrm{mN} / \mathrm{m}$ and $\eta \simeq 1$ Pa.s. By roughly estimating the liquid fraction of the foam before and after imbibitions, we find that the foam is diluted by a factor 2 leading to a viscosity of the order of $\eta \simeq 2$ Pa.s, in agreement with the value obtained from the fit. This value of $\eta$ is intermediate between the viscosity of the dyed solution $\left(10^{-3} \mathrm{~Pa} . \mathrm{s}\right)$ and the one of the continuous phase $\left(5 \times 10^{2}\right.$ Pa.s). This is likely due to the fact that typical time for mixing the foam liquid and the dyed water in the channels is of the order of a few hundred of seconds.

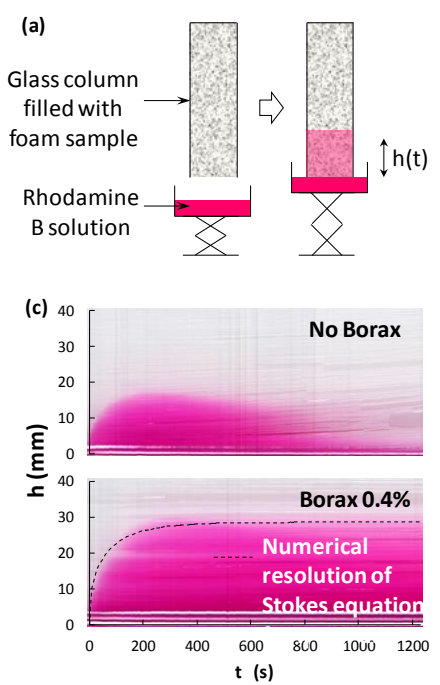

(b)

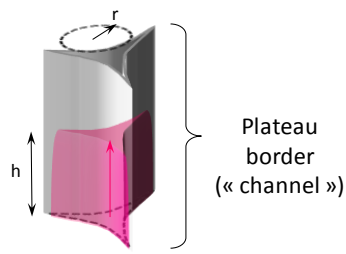

Figure 4. Imbibition properties of the gellified foams. a) Schematic view of the imbibitions experiment. b) Schematic drawing of the dyed liquid rising in a Plateau border, or foam channel, with radius of curvature $r$. c) Space-time plot on a vertical line showing the height $h$ reached by the dyed liquid rising into the foam as a function of time. For the foam without Borax (top), the height decreases after 300 seconds as the foam collapses, while for the 0.4 wt\% Borax containing foam (bottom), the dyed liquid is stored into the foam for hours. The dashed line represents the numerical resolution of Equation (3) for $\eta=1$ Pa.s $(r=150 \mu \mathrm{m}$, and $\gamma=$ $42 \mathrm{mN} / \mathrm{m}$ ).

This has a crucial consequence - in the same foam, the capillary rise can be faster by orders of magnitude than the drainage flow, thus leading to the possibility of using these highly stable foams to suck liquid of low viscosity. These foams are therefore good candidates for decontamination applications such as in nuclear experiments, where contaminants have to be removed from solid surfaces and treated afterward. As an illustration, Figure $\mathbf{5}$ shows that when a piece of foam is put into contact with a dyed liquid, it quickly absorbs it. The 'loaded' foam can then be easily dried and compressed to be stored. (a)

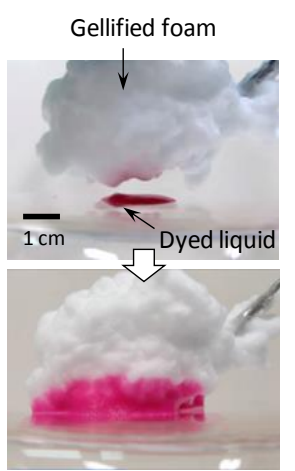

(b)

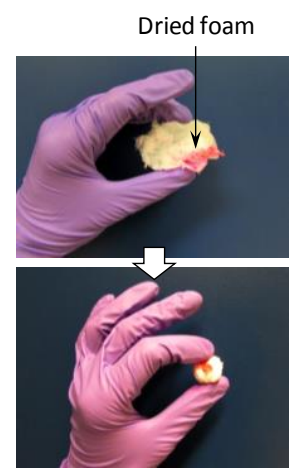

Figure 5. Illustration of the potential of the gel foams for cleaning or decontamination applications. a) A piece of foam is put into contact with a dyed liquid spilled on surface and absorbs the liquid. b) the foam can be dried afterwards and easily compressed manually so that the volume occupied by the 'contaminated' material is low.

\section{Conclusion}

As a conclusion we provide for the first time a method to produce large volumes of highly stable gellified foams consisting in a twostep process, where a polymer solution, PVA, is first foamed using a high shear, and then a cross-linker, Borax, is added to this foam under continuous shearing. This two-step method could be easily applied to other types of foaming processes and other polymer/cross-linker systems. In these foams the PVA molecules are highly cross-linked both in the bulk phase and at the interface. Indeed modeling the drainage flow we find that the drainage time is consistent with very large surface viscosities of the adsorbed polymer layer meaning that the stability of these foams is controlled by large viscosities, both at interfaces and in the bulk. Lastly, we show that these foams are good candidates for cleaning or decontamination processes as they quickly absorb liquids with a typical imbibition time orders of magnitude faster than the drainage rate. The loaded foams can be easily dried and stored imbibition.

\section{Acknowledgements}

The authors thank T. Narita for useful discussions about PVA/Borax systems, G. Ducouret for her help in rheological measurements, as well as A. Colin for discussions on foam drainage

\section{References}

1. Banhart, J. Metal Foams: Production and Stability. Adv. Eng. Mater. 8, 781-794 (2006).

2. Schüler, F. et al. Synthesis of Macroporous Polystyrene by the Polymerization of Foamed Emulsions. Angew. Chem. Int. Ed. 51, 2213-2217 (2012).

3. Pierre, J., Dollet, B. \& Leroy, V. Resonant Acoustic Propagation and Negative Density in Liquid Foams. Phys. Rev. Lett. 112, (2014). 
4. Scheffler, M., Colombo, P. \& Wiley InterScience (Online service). Cellular ceramics structure, manufacturing, properties and applications. (Wiley-VCH ; John Wiley [distributor], 2005). at <http://www.123library.org/book_details/?id=29149>

5. Dame, C., Fritz, C., Pitois, O. \& Faure, S. Relations between physicochemical properties and instability of decontamination foams. Colloids Surf. Physicochem. Eng. Asp. 263, 210-218 (2005).

6. Gurau, D. \& Deju, R. The use of chemical gel for decontamination during decommissioning of nuclear facilities. Radiat. Phys. Chem. 106, 371-375 (2015).

7. Piroird, K. \& Lorenceau, É. Capillary Flow of Oil in a Single Foam Microchannel. Phys. Rev. Lett. 111, (2013).

8. Saint-Jalmes, A. Physical chemistry in foam drainage and coarsening. Soft Matter 2, 836 (2006).

9. Cohen-Addad, S., Höhler, R. \& Pitois, O. Flow in Foams and Flowing Foams. Annu. Rev. Fluid Mech. 45, 241-267 (2013).

10. Surfactant foams doped with laponite: unusual behaviors induced by aging and confinement.

11. Lesov, I., Tcholakova, S. \& Denkov, N. Factors controlling the formation and stability of foams used as precursors of porous materials. J. Colloid Interface Sci. 426, 9-21 (2014).

12. Andersen, T., Melvik, J. E., Gåserød, O., Alsberg, E. \& Christensen, B. E. Ionically Gelled Alginate Foams: Physical Properties Controlled by Operational and Macromolecular Parameters. Biomacromolecules 13, 3703-3710 (2012).

13. Andersen, T., Melvik, J. E., Gåserød, O., Alsberg, E. \& Christensen, B. E. lonically gelled alginate foams: Physical properties controlled by type, amount and source of gelling ions. Carbohydr. Polym. 99, 249-256 (2014).

14. Barbetta, A., Rizzitelli, G., Bedini, R., Pecci, R. \& Dentini, M. Porous gelatin hydrogels by gas-in-liquid foam templating. Soft Matter 6, 1785 (2010).

15. Colosi, C. et al. Morphological Comparison of PVA Scaffolds Obtained by Gas Foaming and Microfluidic Foaming Techniques. Langmuir 29, 82-91 (2013).

16. Testouri, A., Honorez, C., Barillec, A., Langevin, D. \& Drenckhan, W. Highly Structured Foams from Chitosan Gels. Macromolecules 43, 6166-6173 (2010).

17. Schultz, R. K. \& Myers, R. R. The Chemorheology of Poly(vinyl alcohol)-Borate Gels. Macromolecules 2, 281-285 (1969).

18. Pezron, E., Leibler, L., Ricard, A. \& Audebert, R. Reversible gel formation induced by ion complexation. 2. Phase diagrams. Macromolecules 21, 1126-1131 (1988).

19. Lifshitz, I. M. \& Slyozov, V. V. The kinetics of precipitation from supersaturated solid solutions. J. Phys. Chem. Solids 19, 35-50 (1961).

20. Wagner, $\mathrm{C}$. Theorie der Alterung von Niederschlägen durch Umlösen (Ostwald-Reifung). Z. Für Elektrochem. Berichte Bunsenges. Für Phys. Chem. 65, 581-591 (1961).

21. Kloek, W., van Vliet, T. \& Meinders, M. Effect of Bulk and Interfacial Rheological Properties on Bubble Dissolution. J. Colloid Interface Sci. 237, 158-166 (2001).

22. Koehler, S. A., Hilgenfeldt, S. \& Stone, H. A. A Generalized View of Foam Drainage: Experiment and Theory. Langmuir 16, 6327-6341 (2000).

23. Carrier, V., Destouesse, S. \& Colin, A. Foam drainage: A film contribution? Phys. Rev. E 65, (2002).

24. Lorenceau, E., Louvet, N., Rouyer, F. \& Pitois, O. Permeability of aqueous foams. Eur. Phys. J. E 28, 293-304 (2009).
25. Saint-Jalmes, A. \& Langevin, D. Time evolution of aqueous foams: drainage and coarsening. J. Phys. Condens. Matter 14, 9397-9412 (2002).

26. Nguyen, A. V. Liquid Drainage in Single Plateau Borders of Foam. J. Colloid Interface Sci. 249, 194-199 (2002).

27. Maerker, J. M. Rheology Resulting from Shear-Induced Structure in Associating Polymer Solutions. J. Rheol. 30, 77 (1986).

28. Vandebril, S., Franck, A., Fuller, G. G., Moldenaers, P. \& Vermant, J. A double wall-ring geometry for interfacial shear rheometry. Rheol. Acta 49, 131-144 (2010).

29. Maestro, A. et al. Reptation in langmuir polymer monolayers. Soft Matter 6, 4407 (2010).

30. Spigone, E., Cho, G.-Y., Fuller, G. G. \& Cicuta, P. Surface Rheology of a Polymer Monolayer: Effects of Polymer Chain Length and Compression Rate. Langmuir 25, 7457-7464 (2009).

31. Monteux, C. Adsorption of soluble polymers at liquid interfaces and in foams. Comptes Rendus Phys. 15, 775-785 (2014).

32. Mensire, R., Piroird, K. \& Lorenceau, E. Coalescence of dry foam under water injection. Soft Matter 10, 7068-7072 (2014).

33. Washburn, E. W. The Dynamics of Capillary Flow. Phys. Rev. 17, 273-283 (1921). 